\title{
Sleep quality and glycaemic variability in a real-life setting in adults with type 1 diabetes
}

\author{
Rachel Brandt ${ }^{1} \cdot$ Minsun Park $^{2} \cdot$ Kristen Wroblewski $^{3} \cdot$ Lauretta Quinn $^{2} \cdot$ Esra Tasali $^{4} \cdot$ Ali Cinar $^{5}$ (D) \\ Received: 4 January 2021 / Accepted: 24 March 2021 / Published online: 17 June 2021 \\ (C) The Author(s), under exclusive licence to Springer-Verlag GmbH Germany, part of Springer Nature 2021
}

\begin{abstract}
Aims/hypothesis Suboptimal subjective sleep quality is very common in adults with type 1 diabetes. Reducing glycaemic variability is a key objective in this population. To date, no prior studies have examined the associations between objectively measured sleep quality and overnight glycaemic variability in adults with type 1 diabetes. We aimed to test the hypothesis that poor sleep quality would be associated with greater overnight glycaemic variability.

Methods Data were collected in the home setting from 20 adults (ten male and ten female participants) with type 1 diabetes who were current insulin pump users. Simultaneous assessments of objective sleep quality (Zmachine Insight+) and continuous glucose monitoring (CGM) were performed over multiple nights (up to 15 nights) in each participant. Due to the real-life nature of this study, the participants kept their usual CGM alerts for out-of-range glucose values. Sleep quality was categorised as 'good' or 'poor' using a composite of objective sleep features (i.e. sleep efficiency, wake after sleep onset and number of awakenings) based on the National Sleep Foundation's consensus criteria. Glycaemic variability was quantified using SD and CV of overnight glucose values based on overnight CGM profiles.

Results A total of 170 nights were analysed. Overall, 86 (51\%) nights were categorised as good sleep quality, and 84 (49\%) nights were categorised as poor sleep quality. Using linear mixed-effects models, poor sleep quality was significantly associated with greater glycaemic variability as quantified by SD (coefficient: 0.39 [95\% CI 0.10, 0.67], $p=0.009$ ) and CV (coefficient: 4.35 [95\% CI 0.8, 7.9], $p=0.02$ ) of overnight glucose values, after accounting for age, sex, BMI and overnight insulin dose. There was large inter- and intra-individual variability in sleep and glycaemic characteristics. Both pooled and individual-level data revealed that the nights with poor sleep quality had larger SDs and CVs, and, conversely, the nights with good sleep quality had smaller SDs and CVs. No associations were found between sleep quality and time spent in the target glucose range, or above or below the target glucose range, where CGM alarms are most likely to occur.

Conclusions/interpretation Objectively measured sleep quality is associated with overnight glycaemic variability in adults with type 1 diabetes. These findings highlight an important role of sleep quality in overnight glycaemic control in type 1 diabetes. They also provide a strong incentive to both patients and healthcare providers for considering sleep quality in personalised type 1 diabetes glycaemic management plans. Future studies should investigate the mechanisms linking sleep quality to glycaemic variability.
\end{abstract}

Keywords Glycaemic variability $\cdot$ Sleep $\cdot$ Sleep quality $\cdot$ Type 1 diabetes

\section{Abbreviations \\ CGM Continuous glucose monitoring \\ NREM Non-rapid eye movement}

REM Rapid eye movement

WASO Wake after sleep onset

Esra Tasali, Lauretta Quinn and Ali Cinar are co-senior authors.

Ali Cinar

cinar@iit.edu

1 Department of Biomedical Engineering, Illinois Institute of Technology, Chicago, IL, USA

2 College of Nursing, University of Illinois at Chicago, Chicago, IL, USA
3 Department of Public Health Sciences, University of Chicago, Chicago, IL, USA

4 Department of Medicine, University of Chicago, Chicago, IL, USA

5 Department of Chemical and Biological Engineering, Illinois Institute of Technology, Chicago, IL, USA 


\section{Research in context}

\section{What is already known about this subject?}

- Suboptimal subjective sleep quality is very common in people with type 1 diabetes

- Reducing glycaemic variability is a key objective in glycaemic management in type 1 diabetes

\section{What is the key question?}

- Is objectively measured sleep quality associated with overnight glycaemic variability in adults with type 1 diabetes?

\section{What are the new findings?}

- In a real-life setting, there was a large inter- and intra-individual variability in objective sleep and glycaemic characteristics in adults with type 1 diabetes

- Both pooled and individual-level data revealed that the nights with poor sleep quality had greater glycaemic variability, and, conversely, the nights with good sleep quality had smaller glycaemic variability, after accounting for age, sex, BMI and overnight insulin dose

- Objectively measured sleep quality is associated with overnight glycaemic variability in adults with type 1 diabetes after controlling for traditional factors

\section{How might this impact on clinical practice in the foreseeable future?}

- Sleep quality has an important role in overnight glycaemic control in type 1 diabetes. These findings provide a strong incentive to both patients and healthcare providers for considering sleep quality in personalised type 1 diabetes glycaemic management plans

\section{Introduction}

In type 1 diabetes, multiple factors need to be considered in achieving goals for optimal blood glucose control. One of these factors that has been increasingly acknowledged is sleep. Over the past two decades, substantial evidence has accumulated highlighting the importance of sleep in glucose regulation [1]. Only recently has the American Diabetes Association's Standards of Medical Care recommended assessment of sleep patterns as part of comprehensive medical evaluation [2].

Sleep quality is a well-recognised predictor of health and is multidimensional in nature [3]. Sleep quality can be measured by self-ratings of 'good' or 'poor' sleep, which signify 'subjective sleep quality', or by sleep monitors (e.g. actigraphy or polysomnography), which signify 'objective sleep quality'. Although self-reported sleep measures are frequently used, they are prone to over- or underestimation of sleep as compared with objective assessments [4]. Among people with type 1 diabetes, suboptimal subjective sleep quality is very common $[1,5]$. Both biological and psychosocial factors can affect sleep quality in this patient population. In turn, poor sleep quality can negatively impact glucose metabolism, as evidenced by experimental disruptions of sleep quality in healthy individuals $[6,7]$.

Glycaemic variability, i.e. fluctuations of glucose over a given time interval, is a fundamental component of glycaemic control $[8,9]$. Particularly in people with type 1 diabetes, reducing glycaemic variability is a key objective in glycaemic management [10]. To date, no prior studies have examined the associations between objectively measured sleep quality and overnight glycaemic variability in adults with type 1 diabetes. We hypothesised that poor sleep quality would be associated with greater overnight glycaemic variability in type 1 diabetes. To test this hypothesis, we analysed data collected in the home setting over multiple nights (up to 15 nights), using objective sleep assessments and continuous glucose monitoring (CGM), in adults with type 1 diabetes.

\section{Methods}

Participants Data were collected as part of two clinical research studies of adults with type 1 diabetes conducted at the University of Illinois at Chicago and Illinois Institute of Technology. Both studies were observational with a single group and non-randomised design, and data were collected during sleep or other activities of daily living. The study protocols were approved by the Institutional Review Boards and participants gave informed consent. Eligible participants were adults between the ages of 18 and 60 years, who were diagnosed with type 1 diabetes (physician diagnosis with at least 1 year of disease duration) and who were current insulin pump users. Participants were not screened for any sleep disorders. 
Participants were excluded if they had history of significant cardiovascular disease, nephropathy, severe retinopathy, severe neuropathy, or significant musculoskeletal or other systemic chronic disease. Participants were also excluded if they had had any episodes of severe hypoglycaemia (i.e. episode requiring assistance from another person to treat and/or hospitalisation) or diabetic ketoacidosis in the last month before study enrolment. All participants from both studies who had completed simultaneous at home sleep recordings and CGM were included.

Home sleep assessments and CGM All participants were asked to wear a CGM device and a sleep monitor simultaneously at home while following their habitual bedtime and daily routines. Each participant wore the same set of devices throughout the study. In the first study protocol, participants were asked to wear both monitors over a 2 week period, i.e. a total of 14 nights. In the second study protocol, participants were asked to wear both monitors only during the week nights three times over a 2 month period, i.e. a total of 15 nights. Due to the reallife nature of this study, the participants kept their usual CGM alerts for out-of-range glucose values. The CGM device models were Dexcom G4, Dexcom G5 and Dexcom G6 (Dexcom, San Diego, CA, USA). All CGM devices measured glucose levels every $5 \mathrm{~min}$. The sleep monitor (Zmachine Insight+; General Sleep, Cleveland, OH, USA) was a single-channel, EEG-based objective sleep measurement system for in-home use. The EEG electrodes were worn on each mastoid. Sleep-wake stages were determined by an algorithm every $30 \mathrm{~s}$ using frequency and time domain EEG features, which has been validated against the gold standard polysomnography [11]. The computed sleep metrics include total sleep time, sleep efficiency, sleep latency, wake after sleep onset (WASO) and sleep stages, i.e. light nonrapid eye movement (NREM), deep NREM and rapid eye movement (REM) sleep.

Sleep quality We used a composite of objective sleep features based on the National Sleep Foundation's consensus recommendations for sleep quality assessment [3]. Sleep quality was categorised as 'poor' if at least two of the following three criteria were met: (1) sleep efficiency $<85 \%$; or (2) WASO $>40 \mathrm{~min}$; or (3) number of awakenings $>4$; otherwise, sleep quality was categorised as 'good'. Sleep efficiency was defined as the percentage of time spent asleep during time in bed. WASO was defined as the time in minutes spent awake after sleep onset and before the final awakening. Number of awakenings was defined as the number of awake periods $>5$ min after sleep onset. Additionally, we used the time in minutes spent in light NREM, deep NREM and REM sleep stages as indicators of sleep architecture [3].

Overnight glycaemic variability metrics Overnight CGM profiles were constructed by extracting the glucose values during the bedtime interval as recorded by the Zmachine Insight+. Overnight glycaemic variability was quantified by computing the SD and CV of overnight glucose values for each night in each participant. CV was calculated as $(\mathrm{SD} / \mathrm{mean}) \times 100 \%$. While there are several metrics of glycaemic variability, we selected the SD and CV because they are traditional measures of short-term excursions in glucose concentrations and they show high correlations with other glycaemic metrics [8, 9]. Also, the $\mathrm{CV}$ is a clinical care metric that normalises for differences in mean glucose level [10]. Additionally, we have assessed overnight glucose dynamics using other standardised CGM metrics for clinical care [10]. The percentage of time spent with glucose between 3.9 and $10 \mathrm{mmol} / \mathrm{l}$, i.e. time in the target range; the percentage of time spent with glucose $>10 \mathrm{mmol} / \mathrm{l}$, i.e. time above the target range; and the percentage of time spent with glucose $<3.9 \mathrm{mmol} / 1$, i.e. time below the target range, were calculated during each night in each participant.

Statistical analysis Linear mixed-effects models were developed using MATLAB 2016a software (MathWorks, Natick, MA, USA) to examine the relationships between metrics of sleep quality and overnight glycaemic variability. This type of model accounts for correlations between multiple observations per participant [12]. The response variables were SD of overnight glucose; CV of overnight glucose; and the percentage of time spent in, above and below the target glucose range. In the models, sleep quality (categorical variable; 'poor' vs 'good'); minutes spent in light NREM, deep NREM and REM sleep; age; sex; BMI; and overnight insulin dose were considered as fixed effects, while the participants were considered as a random effect. The covariates, i.e. age, sex, BMI and total overnight insulin dose, were selected based on their known influence on both sleep and glycaemic variables [13-15]. Model variables with $p$ values $<0.05$ were considered statistically significant.

All available data from each participant with simultaneous recordings by CGM and Zmachine Insight+ were considered for inclusion in the analysis. To ensure adequate data quality, we applied set criteria to the entire dataset prior to analysis. Nights were excluded from the analysis if any of the following criteria were met: (1) invalid recording due to a technical failure of Zmachine Insight+; (2) total recording time of less than $4 \mathrm{~h}$; (3) total sleep time of less than $2 \mathrm{~h}$; (4) overnight CGM profiles with more than $30 \mathrm{~min}$ of missing data. Based on these criteria, a total of 48 nights $(22 \%)$ were excluded from a total of 218 nights that were recorded. This resulted in a total of 170 nights $(78 \%)$ from a total of 20 participants being included in the final analysis (electronic supplementary material [ESM] Table 1).

\section{Results}

Participant characteristics Participants were ten male and ten female individuals (Table 1). The age range was between 18 
Table 1 Participant characteristics

\begin{tabular}{ll}
\hline Characteristic & All participants $(n=20)$ \\
\hline Age, years & $30.0 \pm 10.4$ \\
Male, $\mathrm{n}(\%)$ & $10(50)$ \\
BMI, $\mathrm{kg} / \mathrm{m}^{2}$ & $28.0 \pm 6.9$ \\
Ethnicity, $\mathrm{n}(\%)$ & \\
$\quad$ African-American & $3(15)$ \\
Asian & $0(0)$ \\
White & $16(80)$ \\
Hispanic & $1(5)$ \\
Systolic BP, mmHg & \\
Diastolic BP, $\mathrm{mmHg}^{\mathrm{a}}$ & $120 \pm 10.5$ \\
$\mathrm{HbA}_{1 \mathrm{c}}, \mathrm{mmol} / \mathrm{mol}^{\mathrm{a}}$ & $75 \pm 8.0$ \\
$\mathrm{HbA}_{1 \mathrm{c}}, \%$ & $48.1 \pm 7.8$ \\
Duration of diabetes, years & $6.6 \pm 0.7$ \\
\hline
\end{tabular}

Data are presented as mean \pm SD unless otherwise specified

${ }^{\text {a }}$ Data available in $n=16$ participants

and 57 years. Among all participants, ten had a BMI in the normal range, five were overweight and another five were obese. The $\mathrm{HbA}_{1 \mathrm{c}}$ values ranged from $40 \mathrm{mmol} / \mathrm{mol}(5.8 \%)$ to $68 \mathrm{mmol} / \mathrm{mol}(8.4 \%)$, with 16 out of 20 participants having an $\mathrm{HbA}_{1 \mathrm{c}}<53 \mathrm{mmol} / \mathrm{mol}(7 \%)$.

Sleep and overnight glycaemic characteristics Overall, 86 (51\%) nights were categorised as good sleep quality, and 84 (49\%) nights were categorised as poor sleep quality (Table 2). Out of a total of 170 nights, $112(66 \%)$ met at least one out of three criteria, $84(49 \%)$ met at least two out of three criteria and $51(30 \%)$ met three out of three criteria for poor sleep quality. Sleep characteristics showed large differences between individuals and marked intra-individual variability (Table 2). Considering all nights, on average, the sleep efficiency was $\sim 79 \%$ (ranging from $43 \%$ to $98 \%$ ), WASO was $\sim 73 \mathrm{~min}$ (ranging from 7 to $324 \mathrm{~min}$ ) and number of awakenings was $\sim 3$ (ranging from 0 to 18 ). On average, the participants slept $6.0 \pm 1.6 \mathrm{~h}$, with $203.1 \pm 58.7 \mathrm{~min}$ spent in light NREM sleep, $70.0 \pm 37.8$ min spent in deep NREM sleep and $85.0 \pm 42.7$ min spent in REM sleep.

Similar to sleep characteristics, there was substantial interand intra-individual variability in overnight glycaemic characteristics (Table 3). Considering all nights, on average, the mean overnight glucose level was $\sim 7.9 \mathrm{mmol} / \mathrm{l}$ (ranging from 3.3 to $17.1 \mathrm{mmol} / \mathrm{l}$ ), SD of overnight glucose was $\sim 1.4 \mathrm{mmol} / 1$ (ranging from 0.11 to $5.3 \mathrm{mmol} / \mathrm{l}$ ) and $\mathrm{CV}$ of overnight glucose was $\sim 19 \%$ (ranging from $2 \%$ to $62 \%$ ). Overall, the time spent in the target glucose range was $\sim 68 \%$, the time spent above the target glucose range was $\sim 25 \%$ and the time spent below the target glucose range was only $\sim 7 \%$.
Associations between sleep quality and glycaemic variability Poor sleep quality was significantly associated with greater glycaemic variability as quantified by the SD (coefficient: 0.39 [95\% CI 0.10, 0.67], $p=0.009$ ) and CV of overnight glucose values (coefficient: 4.35 [95\% CI 0.8, 7.9], $p=0.02$ ) after accounting for age, sex, BMI and overnight insulin dose (Table 4). Both the pooled sample (Figs 1a, 2a) and individual-level (Figs 1b, 2b) data revealed that the nights with poor sleep quality had larger SDs and CVs, and, conversely, the nights with good sleep quality had smaller SDs and CVs. We did not find statistically significant relationships between sleep duration or minutes spent in light NREM, deep NREM or REM sleep and glycaemic variability metrics, after accounting for age, sex, BMI and overnight insulin dose (ESM Tables 2, 3). The sleep efficiency and WASO were significantly associated with the $\mathrm{CV}$ and SD of overnight glucose values after accounting for age, sex, BMI and overnight insulin dose (ESM Table 4). There were no significant associations between sleep quality and percentage of time spent above, below or in the target glucose range after accounting for age, sex, BMI and overnight insulin dose (Table 4). There were no significant associations between WASO or number of awakenings and the time spent above or below the target glucose range (ESM Table 5). The numbers of nights with any time spent above and/or below the target glucose range were similar between good and poor sleep quality nights. Out of a total of 170 nights that were analysed, a total of 50 nights showed time spent below the target glucose range. Of those 50 nights, 24 were categorised as good sleep quality and 26 were categorised as poor sleep quality. Additionally, a total of 92 out of 170 nights showed time spent above the target glucose range. Of those 92 nights, 43 were categorised as good sleep quality and 49 were categorised as poor sleep quality (ESM Tables 6, 7).

\section{Discussion}

We have demonstrated that in adults with type 1 diabetes, objectively assessed sleep quality is significantly associated with overnight glycaemic variability, after controlling for age, sex, BMI and insulin use. Overall, the nights with poor sleep quality had larger overnight glycaemic variability, and conversely the nights with good sleep quality had smaller overnight glycaemic variability, after accounting for traditional factors that are known to influence both sleep and blood glucose levels. These findings highlight an important role of sleep quality in overnight glycaemic control in type 1 diabetes. Moreover, they provide a strong incentive to both patients and healthcare providers to consider sleep quality to guide treatment decisions in type 1 diabetes.

To our knowledge, this is the first study to investigate the relationships between objective sleep quality and overnight 
Table 2 Sleep characteristics in individual participants

\begin{tabular}{|c|c|c|c|c|c|c|c|}
\hline \multirow[t]{2}{*}{ Participant } & \multicolumn{3}{|c|}{ Number of nights } & \multirow[t]{2}{*}{ Total sleep time (h) } & \multirow[t]{2}{*}{ Sleep efficiency (\%) } & \multirow[t]{2}{*}{ WASO (min) } & \multirow[t]{2}{*}{ Number of awakenings $\mathrm{s}^{\mathrm{b}}$} \\
\hline & Total & Good sleep quality ${ }^{\mathrm{a}}$ & Poor sleep quality ${ }^{\mathrm{a}}$ & & & & \\
\hline 1 & 12 & 8 & 4 & $5.9 \pm 1.2$ & $85.1 \pm 7.4$ & $50.7 \pm 26.2$ & $2.5 \pm 1.5$ \\
\hline 2 & 13 & 11 & 2 & $7.4 \pm 1.3$ & $90.8 \pm 3.6$ & $34.0 \pm 14.5$ & $1 \pm 1$ \\
\hline 3 & 15 & 13 & 2 & $6.5 \pm 0.6$ & $89.3 \pm 2.8$ & $35.0 \pm 9.1$ & $0.7 \pm 0.8$ \\
\hline 4 & 13 & 8 & 5 & $7.4 \pm 0.5$ & $82.2 \pm 5.4$ & $53.7 \pm 18.6$ & $1.6 \pm 1.3$ \\
\hline 5 & 3 & 3 & 0 & $7.6 \pm 1.8$ & $84.7 \pm 4.1$ & $29.7 \pm 5.5$ & $0.5 \pm 0.5$ \\
\hline 6 & 10 & 3 & 7 & $6.0 \pm 1.4$ & $76.9 \pm 11.1$ & $73.8 \pm 37.4$ & $2.6 \pm 1.5$ \\
\hline 7 & 12 & 6 & 6 & $5.2 \pm 2.0$ & $68.8 \pm 27.6$ & $130.3 \pm 119.1$ & $4.6 \pm 5.4$ \\
\hline 8 & 10 & 5 & 5 & $5.9 \pm 1.9$ & $76.8 \pm 22.1$ & $98.2 \pm 96.3$ & $4.8 \pm 6.0$ \\
\hline 9 & 9 & 0 & 9 & $5.4 \pm 0.6$ & $66.5 \pm 5.6$ & $124.4 \pm 37.6$ & $6.7 \pm 2.5$ \\
\hline 10 & 5 & 0 & 5 & $5.1 \pm 2.3$ & $60.1 \pm 19.8$ & $171.6 \pm 85.8$ & $9.4 \pm 5.2$ \\
\hline 11 & 4 & 2 & 2 & $6.4 \pm 1.0$ & $83.9 \pm 3.5$ & $57.3 \pm 13.2$ & $1.5 \pm 1$ \\
\hline 12 & 7 & 5 & 2 & $6.8 \pm 0.8$ & $88.3 \pm 17.4$ & $44.8 \pm 35.9$ & $1.6 \pm 2.3$ \\
\hline 13 & 9 & 6 & 3 & $5.5 \pm 1.3$ & $91.3 \pm 6.9$ & $29.0 \pm 24.2$ & $1.3 \pm 1.8$ \\
\hline 14 & 14 & 0 & 14 & $3.5 \pm 1.0$ & $49.1 \pm 17.6$ & $121.1 \pm 38.1$ & $5.5 \pm 2.3$ \\
\hline 15 & 6 & 3 & 3 & $7.2 \pm 0.6$ & $84.2 \pm 3.1$ & $66.6 \pm 18.1$ & $3.3 \pm 1.0$ \\
\hline 16 & 10 & 1 & 9 & $5.5 \pm 1.6$ & $75.7 \pm 8.4$ & $90.2 \pm 39.6$ & $4.2 \pm 1.9$ \\
\hline 17 & 3 & 0 & 3 & $4.3 \pm 1.1$ & $64.6 \pm 12.0$ & $111.2 \pm 37.1$ & $6.3 \pm 1.5$ \\
\hline 18 & 4 & 4 & 0 & $6.4 \pm 1.6$ & $89.4 \pm 1.7$ & $31.0 \pm 15.2$ & $0.75 \pm 1$ \\
\hline 19 & 7 & 6 & 1 & $6.5 \pm 1.6$ & $87.8 \pm 5.3$ & $34.4 \pm 10.6$ & $1.4 \pm 1.6$ \\
\hline 20 & 4 & 2 & 2 & $5.4 \pm 1.4$ & $81.7 \pm 6.1$ & $58.6 \pm 20.1$ & $3.2 \pm 2.2$ \\
\hline Overall & 170 & 86 & 84 & $6.0 \pm 1.6$ & $78.5 \pm 16.4$ & $72.6 \pm 60.0$ & $3.1 \pm 3.3$ \\
\hline
\end{tabular}

Data are presented as mean \pm SD unless otherwise specified

${ }^{a}$ Sleep quality was categorised as 'poor' if at least two of three criteria were met, i.e. sleep efficiency $<85 \%$ or WASO $>40$ min or number of awakenings $>4$, based on the National Sleep Foundation's consensus recommendations for sleep quality assessment

${ }^{\mathrm{b}}$ Number of awakenings was defined as the number of awake periods $>5$ min after sleep onset

glycaemic variability by CGM in adults with type 1 diabetes. Our findings are in agreement with a few studies that found higher overnight glycaemic variability in adults with type 1 diabetes who reported poor sleep quality based on surveys $[16,17]$. In a meta-analysis of adults with type 1 diabetes, some but not all studies found an association between sleep quality and overall glycaemic control as assessed by $\mathrm{HbA}_{1 \mathrm{c}}$ [5]. Adults with optimal sleep quality scores had significantly lower $\mathrm{HbA}_{1 \mathrm{c}}$ based on subjective sleep measures, but not when sleep was objectively assessed [5]. In a recent study of adults with type 1 diabetes, better subjective sleep quality was associated with improved daily blood glucose values [18].

Our finding of greater glycaemic variability with poor sleep quality may have important clinical implications for diabetesrelated complications [8, 19, 20] as well as self-management and adherence behaviours [21]. It has been postulated that insufficient sleep impairs self-care behaviours through impairments of cognitive function and the decision-making process [22]. In support of this concept, a recent study of adults with type 1 diabetes has suggested that self-regulation failure could be an underlying mechanism linking sleep quality to diabetes outcomes [18]. Our study was not designed to investigate the underlying mechanisms (or mediators) of this association, which are likely to be multiple. Nevertheless, physiological evidence from experimental manipulations of sleep in healthy individuals supports the notion that disruptions in sleep quality can be detrimental to glucose metabolism $[6,7]$.

Remarkably, at the individual level, the night-to-night variability in sleep quality was associated with the degree of overnight glycaemic variability in our participants. More specifically, on the nights when a participant had poor sleep quality, the SDs of overnight glucose values were larger, relative to the SDs on the nights with good sleep quality. Similarly, the nights with poor sleep quality were associated with larger CVs of overnight glucose values, indicating that accounting for mean glucose values produced similar results. In fact, nightly variations in sleep patterns are common in the general population, and this intra-individual variability in sleep patterns has been associated with important physical and mental health outcomes [23]. Consistent with our findings, one prior study reported marked night-to-night intraindividual variability in sleep and glycaemic metrics, but the 
Table 3 Overnight glycaemic characteristics in individual participants

\begin{tabular}{|c|c|c|c|c|c|c|c|}
\hline Participant & Number of nights & Mean glucose (mmol/l) & $\mathrm{SD}(\mathrm{mmol} / \mathrm{l})^{\mathrm{a}}$ & $\mathrm{CV}(\%)^{\mathrm{b}}$ & $\begin{array}{l}\text { Time in target } \\
\text { range }(\%)^{\mathrm{c}}\end{array}$ & $\begin{array}{l}\text { Time above } \\
\text { target range }(\%)^{\mathrm{d}}\end{array}$ & $\begin{array}{l}\text { Time below } \\
\text { target range }(\%)^{\mathrm{e}}\end{array}$ \\
\hline 1 & 12 & $7.42 \pm 1.90$ & $1.24 \pm 0.56$ & $18.2 \pm 9.6$ & $85.4 \pm 30.4$ & $13.3 \pm 30.5$ & $1.3 \pm 4.6$ \\
\hline 2 & 13 & $9.85 \pm 2.67$ & $1.55 \pm 0.72$ & $16.6 \pm 7.1$ & $55.3 \pm 37.5$ & $43.9 \pm 38.5$ & $0.8 \pm 2.2$ \\
\hline 3 & 15 & $4.50 \pm 0.55$ & $0.37 \pm 0.19$ & $8.2 \pm 4.1$ & $85.7 \pm 22.0$ & $0 \pm 0$ & $14.3 \pm 22.0$ \\
\hline 4 & 13 & $6.36 \pm 3.25$ & $1.36 \pm 0.80$ & $23.5 \pm 13.9$ & $57.3 \pm 35.1$ & $16.2 \pm 34.3$ & $26.5 \pm 29.9$ \\
\hline 5 & 3 & $8.21 \pm 3.77$ & $0.92 \pm 0.11$ & $14.2 \pm 10.1$ & $53.5 \pm 14.4$ & $31.5 \pm 30.8$ & $14.9 \pm 25.9$ \\
\hline 6 & 10 & $7.41 \pm 1.71$ & $1.27 \pm 0.64$ & $18.4 \pm 6.5$ & $83.9 \pm 24.3$ & $10.8 \pm 22.5$ & $5.3 \pm 13.2$ \\
\hline 7 & 12 & $8.78 \pm 1.93$ & $1.61 \pm 0.58$ & $19.5 \pm 9.8$ & $66.2 \pm 31.9$ & $31.4 \pm 32.9$ & $2.3 \pm 5.5$ \\
\hline 8 & 10 & $5.05 \pm 0.88$ & $0.46 \pm 0.33$ & $8.8 \pm 5.9$ & $97.5 \pm 5.5$ & $0 \pm 0$ & $2.5 \pm 5.5$ \\
\hline 9 & 9 & $10.57 \pm 2.71$ & $1.97 \pm 0.93$ & $18.2 \pm 6.5$ & $47.5 \pm 30.3$ & $52.5 \pm 30.3$ & $0 \pm 0$ \\
\hline 10 & 5 & $7.71 \pm 2.38$ & $2.93 \pm 1.20$ & $38.3 \pm 13.7$ & $60.0 \pm 24.1$ & $28.2 \pm 27.7$ & $11.9 \pm 10.3$ \\
\hline 11 & 4 & $4.49 \pm 0.50$ & $1.52 \pm 0.48$ & $33.9 \pm 11.6$ & $62.1 \pm 16.5$ & $1.0 \pm 2.0$ & $36.9 \pm 18.3$ \\
\hline 12 & 7 & $8.61 \pm 2.25$ & $2.08 \pm 1.74$ & $23.7 \pm 17.6$ & $57.0 \pm 39.6$ & $37.8 \pm 39.1$ & $5.2 \pm 7.3$ \\
\hline 13 & 9 & $10.73 \pm 2.88$ & $1.64 \pm 1.25$ & $16.2 \pm 12.3$ & $42.5 \pm 32.0$ & $57.5 \pm 32.0$ & $0 \pm 0$ \\
\hline 14 & 14 & $8.61 \pm 3.0$ & $1.51 \pm 0.52$ & $19.5 \pm 9.6$ & $65.6 \pm 38.5$ & $30.5 \pm 40.4$ & $3.9 \pm 7.5$ \\
\hline 15 & 6 & $5.30 \pm 0.83$ & $1.54 \pm 0.52$ & $29.1 \pm 8.5$ & $75.6 \pm 17.9$ & $2.2 \pm 3.9$ & $22.2 \pm 17.6$ \\
\hline 16 & 10 & $9.31 \pm 1.97$ & $1.83 \pm 0.93$ & $20.3 \pm 10.2$ & $61.3 \pm 34.9$ & $38.7 \pm 34.9$ & $0 \pm 0$ \\
\hline 17 & 3 & $14.58 \pm 1.81$ & $2.71 \pm 1.83$ & $17.8 \pm 10.1$ & $4.7 \pm 8.1$ & $95.3 \pm 8.1$ & $0 \pm 0$ \\
\hline 18 & 4 & $7.02 \pm 1.09$ & $1.19 \pm 1.21$ & $15.9 \pm 13.9$ & $89.9 \pm 20.3$ & $10.1 \pm 20.3$ & $0 \pm 0$ \\
\hline 19 & 7 & $8.34 \pm 1.57$ & $1.44 \pm 0.76$ & $17.1 \pm 7.2$ & $79.4 \pm 16.4$ & $19.6 \pm 17.5$ & $1.0 \pm 2.7$ \\
\hline 20 & 4 & $9.18 \pm 1.26$ & $1.18 \pm 0.68$ & $12.2 \pm 5.3$ & $74.7 \pm 29.4$ & $25.3 \pm 29.4$ & $0 \pm 0$ \\
\hline Overall & 170 & $7.90 \pm 2.95$ & $1.42 \pm 0.95$ & $18.5 \pm 11.5$ & $67.7 \pm 32.8$ & $25.4 \pm 33.8$ & $6.9 \pm 15.4$ \\
\hline
\end{tabular}

Data are presented as mean \pm SD unless otherwise specified. Glucose levels were assessed using CGM

${ }^{\text {a }} \mathrm{SD}$ of overnight glucose levels

${ }^{\mathrm{b}} \mathrm{CV}$ of overnight glucose levels calculated as $(\mathrm{SD} / \mathrm{mean}) \times 100 \%$

${ }^{\mathrm{c}}$ Time in target range represents the percentage of time spent with glucose between 3.9 and $10 \mathrm{mmol} / \mathrm{l}$

${ }^{\mathrm{d}}$ Time above target range represents the percentage of time spent with glucose $>10 \mathrm{mmol} / \mathrm{l}$

${ }^{\mathrm{e}}$ Time below target range represents the percentage of time spent with glucose $<3.9 \mathrm{mmol} / \mathrm{l}$

role of sleep quality itself in glycaemic variability was not assessed [24]. The authors reported that a higher amount of deep sleep was associated with lower $\mathrm{HbA}_{1 \mathrm{c}}$ levels and less time spent in hypoglycaemia [24]. In our study, there were no significant relationships between time spent in specific sleep stages and overnight glycaemic variability, after controlling for confounding effects of age, sex, BMI and insulin use. We did not find a significant association between sleep quality and percentage of time spent in the target glucose range. One potential explanation for this latter finding could be that $80 \%$ of our participants had overall good glycaemic control, with $\mathrm{HbA}_{1 \mathrm{c}}$ values $<53 \mathrm{mmol} / \mathrm{mol}(7 \%)$.

A major strength of our study is that we performed simultaneous assessments of objective sleep quality and glycaemic variability by CGM. Glycaemic variability has a central role in determining treatment success in type 1 diabetes [10]. Moreover, we collected, for the first time, objective sleep data over multiple nights in adults with type 1 diabetes in the home setting, allowing us to assess intra-individual variability in sleep quality and its impact on glycaemic variability in reallife conditions. Importantly, our statistical models accounted for potential confounding effects of age, sex, BMI and overnight insulin use, all of which are known to influence both sleep and glycaemic metrics.

Our study also has some limitations. We studied a small number of adults with type 1 diabetes with relatively wellcontrolled disease; thus results may not be generalisable to more diverse patient populations such as those with multiple comorbidities and poor glycaemic control. Likewise, our findings cannot be directly translated into children and adolescents with type 1 diabetes because both sleep and glycaemic patterns in these populations are considered to be quite different from adults $[25,26]$. We did not perform formal assessments of sleep disorders (e.g. obstructive sleep apnoea, insomnia), and thus potential confounding from undiagnosed sleep disorders is also possible. Obstructive sleep apnoea is a common comorbidity of type 1 diabetes [5]. A few reports have suggested higher glycaemic variability in non-diabetic 


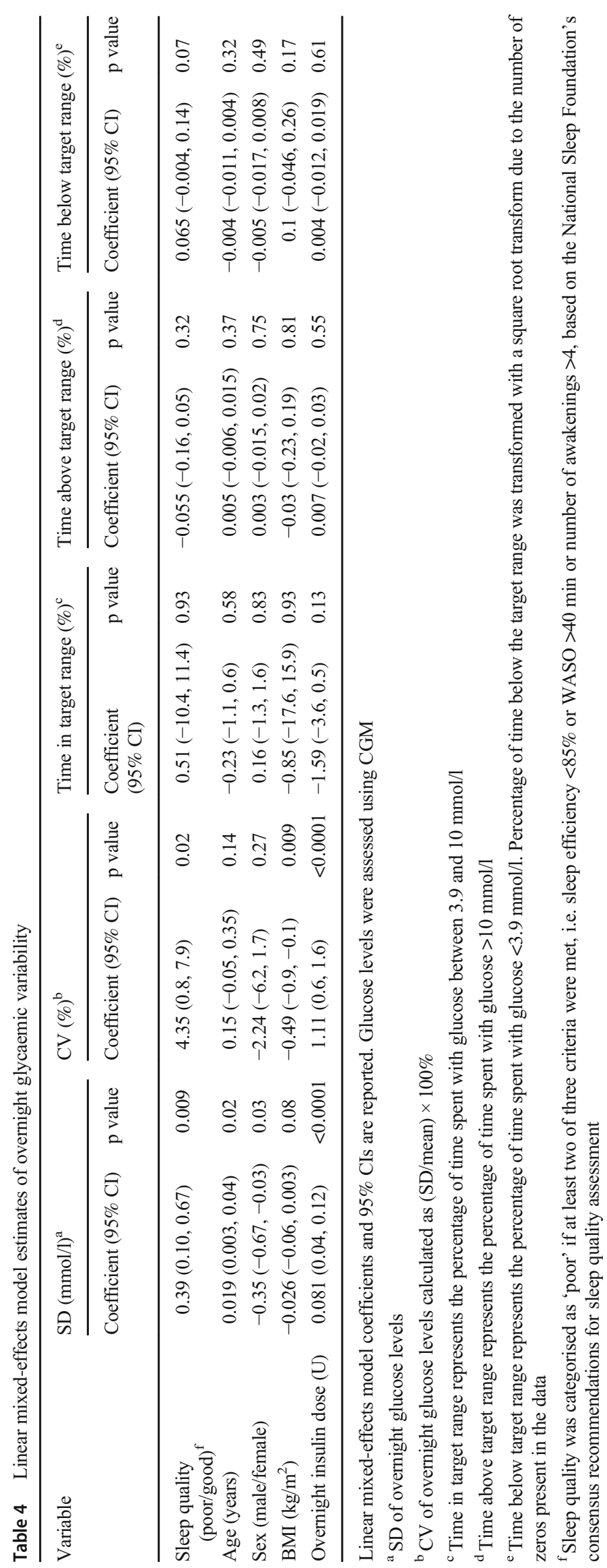


Fig. 1 SD of overnight glucose. (a) Pooled data and (b) individual-level data. Glycaemic variability was derived from the SDs of overnight glucose values using CGM profiles for each participant in each night. SDs of glucose values were adjusted for age, sex, BMI and overnight insulin dose. Blue triangles represent nights with good sleep quality and red circles represent nights with poor sleep quality a

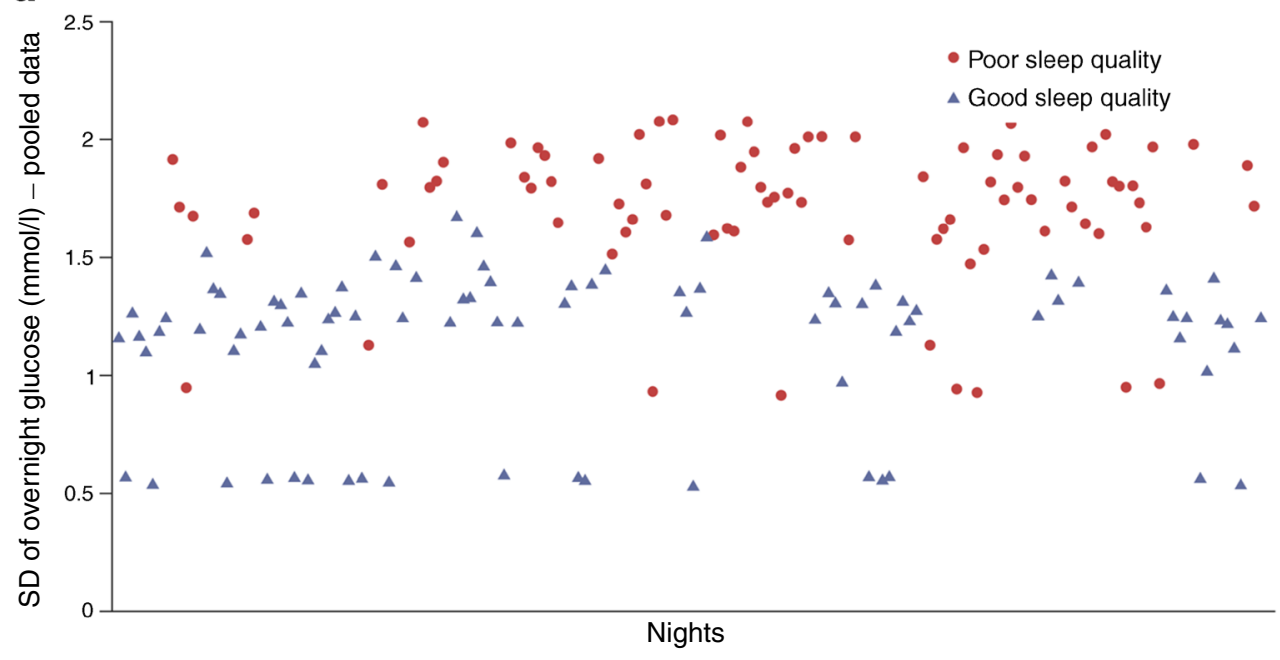

b

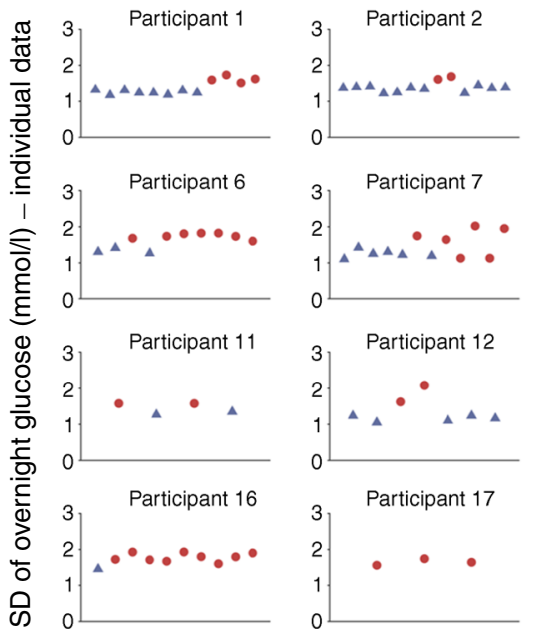

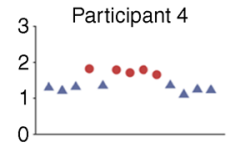
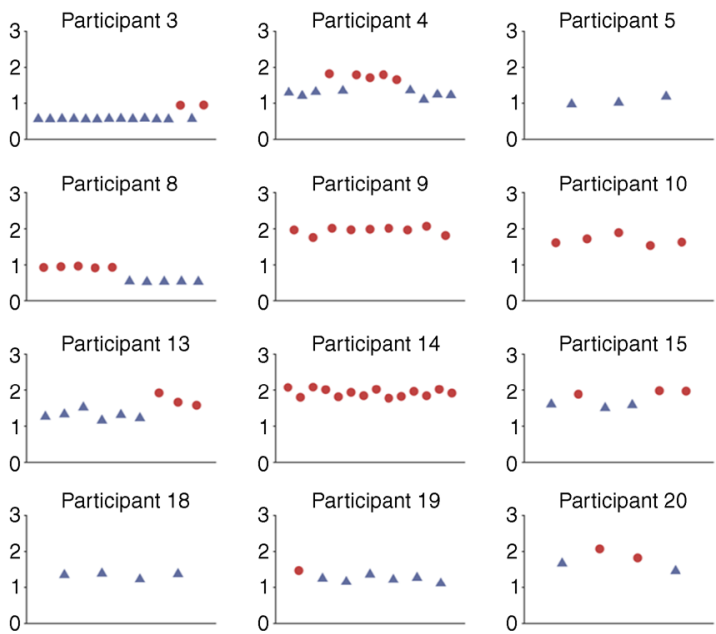

Nights

adults with obstructive sleep apnoea [27, 28]. Being overweight and obesity are major risk factors for obstructive sleep apnoea. In our sample, ten (50\%) participants had a BMI in the obese or overweight range, which makes undiagnosed obstructive sleep apnoea a possible explanation for overall poor sleep quality observed in some of our participants. Glucose fluctuations could be influenced by activities of daily living such as diet and exercise, which were not controlled in our study under real-life conditions [8, 29]. Further, we were not able to examine the impact of week vs weekend nights on sleep quality, because the majority of recordings were collected on weeknights per protocol requirements. Of interest, when visually examined, the night-to-night variability was still apparent even among participants who only had weeknight recordings. Additionally, participants' sleep quality may have been influenced by wearing the devices or CGM alarms. All participants were insulin pump users. To account for a potential impact of pump functions (e.g. predictive low-glucose suspend or closed loop functionality), overnight insulin dose was included as a covariate in our linear mixed-effects model. It is also noteworthy that the CGM alarms were not silenced in our study. The alarms can have varying levels of sound and/or be on vibrate mode depending on the setting by the participants. It is well known that arousals or awakenings from sleep in response to alerts or sounds vary from person to person. Our real-life study was not intended to measure these factors. While it is possible that the CGM alarms may have disrupted sleep quality, we did not find significant associations between sleep quality and time spent above or below the target glucose range, when the alarms are most likely to occur. Additionally, the overall distribution of the number of nights with any time spent above and/or below the target glucose range was similar between the good and poor sleep quality nights. We also acknowledge that the association between sleep and diabetes outcomes is likely bidirectional, in that diabetes itself may also interfere with sleep quality due to multiple reasons (e.g. 
Fig. 2 CV of overnight glucose. (a) Pooled data and (b)

individual-level data. Glycaemic variability was derived from the

CVs of overnight glucose values using CGM profiles for each participant in each night. $\mathrm{CV}$ was calculated as $(\mathrm{SD} /$ mean $) \times 100 \%$. $\mathrm{CVs}$ of glucose values were adjusted for age, sex, BMI and overnight insulin dose. Blue triangles represent nights with good sleep quality and red circles represent nights with poor sleep quality
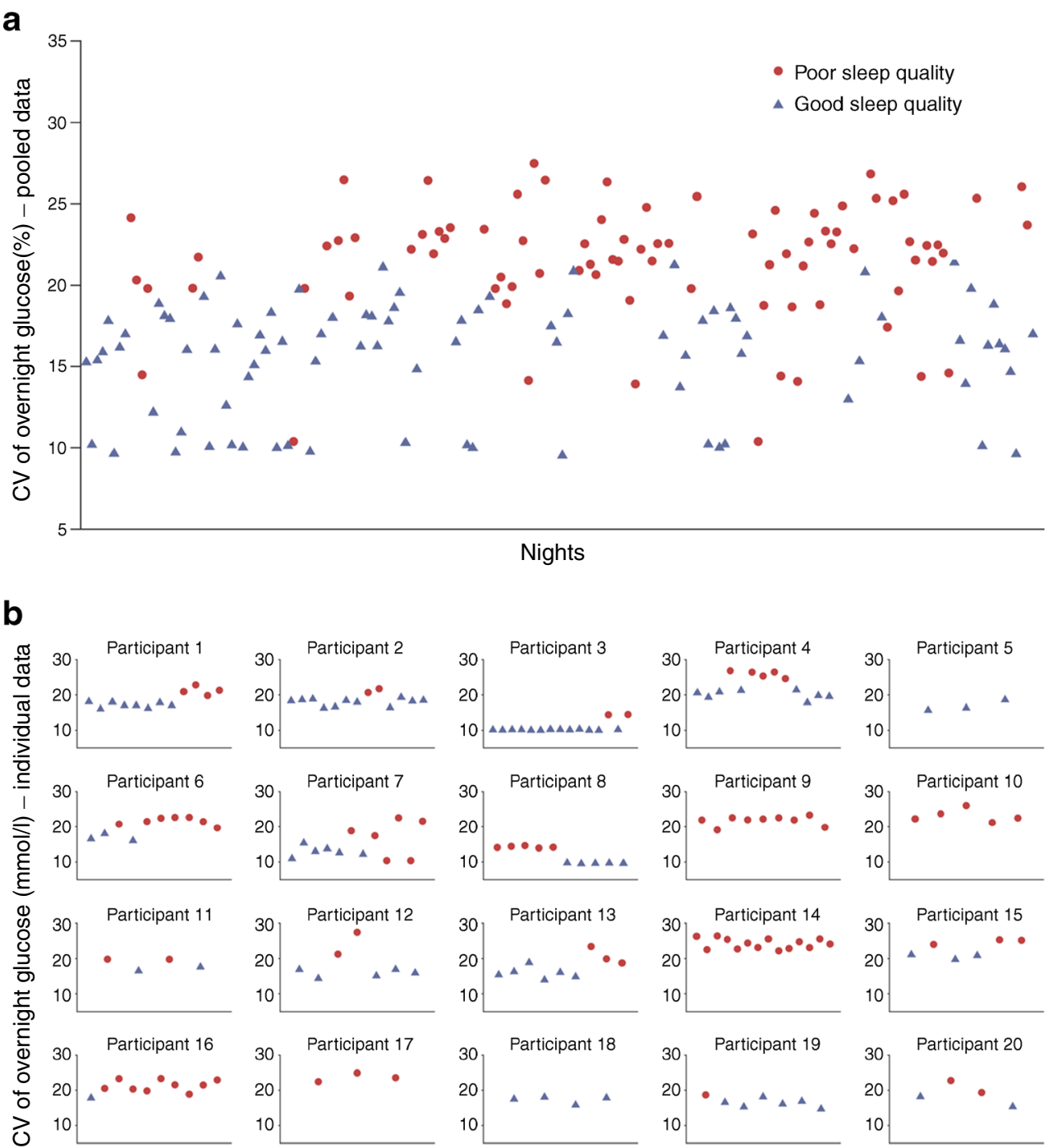
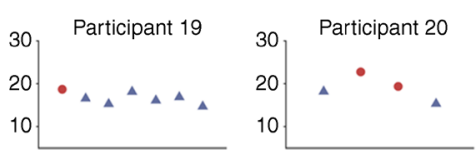

Nights

hypoglycaemia, CGM alarms, frequent urination, neuropathic pain) [1].

In conclusion, we have shown that in adults with type 1 diabetes, objectively measured sleep quality is significantly associated with overnight glycaemic variability. Our findings provide novel insights into the potential role of sleep quality in overnight glycaemic variability in type 1 diabetes, beyond traditional factors. Future research using more rigorous designs, particularly sleep interventions, is warranted to better elucidate the role of sleep quality in glycaemic control in type 1 diabetes. Future studies should also investigate the mechanisms by which sleep quality is associated with glycaemic variability. Additionally, our findings highlight the importance of targeting good sleep quality as part of selfmanagement education for adults with type 1 diabetes. Similarly, healthcare providers should be aware of sleep disruptions and, if present, be able to implement sleep interventions in personalised type 1 diabetes management plans.
Finally, our findings also inform future research into technology for automated insulin delivery systems in type 1 diabetes. Newer type 1 diabetes technology designs should aim to integrate sleep quality in the prediction models and treatment decisions.

Supplementary Information The online version contains peer-reviewed or unedited supplementary material available at https://doi.org/10.1007/ s00125-021-05500-9.

Acknowledgements Funds provided to A. Cinar by the Hyosung S. R. Cho Endowed Chair at Illinois Institute of Technology and scholarships provided to R. Brandt by the ARCS ${ }^{\circledR}$ Foundation's (Achievement Rewards for College Scientists Foundation, Inc.) Illinois Chapter (7/1/ 20-6/20/21) and by the Pritzker Institute of Biomedical Science and Engineering at Illinois Institute of Technology are gratefully acknowledged.

Data availability The datasets generated and analysed during the current studies are not publicly available due to separate ongoing original 
analyses being performed but are available from the corresponding author on reasonable request.

Funding This work was supported by JDRF under grant no. 2-SRA2017-506-M-B made possible through collaboration between JDRF and the Leona M. and Harry B. Helmsley Charitable Trust.

Authors' relationships and activities The authors declare that there are no relationships or activities that might bias, or be perceived to bias, their work.

Contribution statement RB contributed to study design, data acquisition and data analysis and wrote the manuscript. MP contributed to study design and data acquisition and edited the manuscript for intellectual content. ET contributed to data analysis and data interpretation and wrote the manuscript. KW contributed to statistical data analysis and edited the manuscript for intellectual content. LQ and AC obtained funding, designed the study, collected data and edited the manuscript for intellectual content. AC also contributed to data analysis. All authors gave final approval of the version to be published. AC is the guarantor of this work.

\section{References}

1. Nefs GM, Bazelmans E, Donga E, Tack CJ, de Galan BE (2020) Sweet dreams or bitter nightmare: a narrative review of 25 years of research on the role of sleep in diabetes and the contributions of behavioural science. Diabet Med 37(3):418-426. https://doi.org/10. $1111 /$ dme. 14211

2. American Diabetes Association (2020) 4. Comprehensive medical evaluation and assessment of comorbidities: standards of medical care in diabetes-2020. Diabetes Care 43(Suppl 1):S37-S47. https:// doi.org/10.2337/dc20-S004

3. Ohayon M, Wickwire EM, Hirshkowitz M et al (2017) National Sleep Foundation's sleep quality recommendations: first report. Sleep Health 3(1):6-19. https://doi.org/10.1016/j.sleh.2016.11.006

4. O'Donnell D, Silva EJ, Munch M, Ronda JM, Wang W, Duffy JF (2009) Comparison of subjective and objective assessments of sleep in healthy older subjects without sleep complaints. J Sleep Res 18(2):254-263. https://doi.org/10.1111/j.1365-2869.2008. 00719.x

5. Reutrakul S, Thakkinstian A, Anothaisintawee T et al (2016) Sleep characteristics in type 1 diabetes and associations with glycemic control: systematic review and meta-analysis. Sleep Med 23:2645. https://doi.org/10.1016/j.sleep.2016.03.019

6. Tasali E, Leproult R, Ehrmann DA, Van Cauter E (2008) Slowwave sleep and the risk of type 2 diabetes in humans. Proc Natl Acad Sci U S A 105(3):1044-1049. https://doi.org/10.1073/pnas. 0706446105

7. Stamatakis KA, Punjabi NM (2010) Effects of sleep fragmentation on glucose metabolism in normal subjects. Chest 137(1):95-101. https://doi.org/10.1378/chest.09-0791

8. Ceriello A, Monnier L, Owens D (2019) Glycaemic variability in diabetes: clinical and therapeutic implications. Lancet Diabetes Endocrinol 7(3):221-230. https://doi.org/10.1016/S2213-8587(18) 30136-0

9. Rodbard D (2018) Glucose variability: a review of clinical applications and research developments. Diabetes Technol Ther 20(Suppl 2):S25-S215. https://doi.org/10.1089/dia.2018.0092
10. American Diabetes Association (2020) 6. Glycemic targets: standards of medical care in diabetes-2020. Diabetes Care 43(Suppl 1): S66-S76. https://doi.org/10.2337/dc20-S006

11. Kaplan RF, Wang Y, Loparo KA, Kelly MR, Bootzin RR (2014) Performance evaluation of an automated single-channel sleep-wake detection algorithm. Nat Sci Sleep 6:113-122. https://doi.org/10. 2147/NSS.S71159

12. Laird NM, Ware JH (1982) Random-effects models for longitudinal data. Biometrics 38(4):963-974. https://doi.org/10.2307/ 2529876

13. Ohayon MM, Carskadon MA, Guilleminault C, Vitiello MV (2004) Meta-analysis of quantitative sleep parameters from childhood to old age in healthy individuals: developing normative sleep values across the human lifespan. Sleep 27(7):1255-1273. https:// doi.org/10.1093/sleep/27.7.1255

14. Kautzky-Willer A, Harreiter J, Pacini G (2016) Sex and gender differences in risk, pathophysiology and complications of type 2 diabetes mellitus. Endocr Rev 37(3):278-316. https://doi.org/10. 1210/er.2015-1137

15. Mallampalli MP, Carter CL (2014) Exploring sex and gender differences in sleep health: a Society for Women's Health Research report. J Women's Health (Larchmt) 23(7):553-562. https://doi.org/10.1089/jwh.2014.4816

16. Barone MT, Wey D, Schorr F et al (2015) Sleep and glycemic control in type 1 diabetes. Arch Endocrinol Metab 59(1):71-78. https://doi.org/10.1590/2359-3997000000013

17. Martyn-Nemeth P, Phillips SA, Mihailescu D et al (2018) Poor sleep quality is associated with nocturnal glycaemic variability and fear of hypoglycaemia in adults with type 1 diabetes. J Adv Nurs 74(10):2373-2380. https://doi.org/10.1111/jan.13765

18. Tracy EL, Berg CA, Kent De Grey RG et al (2020) The role of selfregulation failures and self-care in the link between daily sleep quality and blood glucose among adults with type 1 diabetes. Ann Behav Med 54(4):249-257. https://doi.org/10.1093/abm/kaz044

19. DeVries JH (2013) Glucose variability: where it is important and how to measure it. Diabetes 62(5):1405-1408. https://doi.org/10. 2337/db12-1610

20. Rodrigues R, de Medeiros LA, Cunha LM et al (2018) Correlations of the glycemic variability with oxidative stress and erythrocytes membrane stability in patients with type 1 diabetes under intensive treatment. Diabetes Res Clin Pract 144:153-160. https://doi.org/10. 1016/j.diabres.2018.01.031

21. Perez KM, Hamburger ER, Lyttle M et al (2018) Sleep in type 1 diabetes: implications for glycemic control and diabetes management. Curr Diab Rep 18(2):5. https://doi.org/10.1007/s11892-0180974-8

22. Riegel B, Weaver TE (2009) Poor sleep and impaired self-care: towards a comprehensive model linking sleep, cognition, and heart failure outcomes. Eur J Cardiovasc Nurs 8(5):337-344. https://doi. org/10.1016/j.ejcnurse.2009.06.003

23. Bei B, Wiley JF, Trinder J, Manber R (2016) Beyond the mean: a systematic review on the correlates of daily intraindividual variability of sleep/wake patterns. Sleep Med Rev 28:108-124. https://doi. org/10.1016/j.smrv.2015.06.003

24. Feupe SF, Frias PF, Mednick SC, McDevitt EA, Heintzman ND (2013) Nocturnal continuous glucose and sleep stage data in adults with type 1 diabetes in real-world conditions. J Diabetes Sci Technol 7(5):1337-1345. https://doi.org/10.1177/ 193229681300700525

25. American Diabetes Association (2020) 13. Children and adolescents: standards of medical care in diabetes-2020. Diabetes Care 43(Suppl 1):S163-S182. https://doi.org/10.2337/dc20-S013 
26. Carskadon MA (2011) Sleep in adolescents: the perfect storm. Pediatr Clin N Am 58(3):637-647. https://doi.org/10.1016/j.pcl. 2011.03.003

27. Peng CS, Cao YA, Tian YH, Zhang WL, Xia J, Yang L (2017) Features of continuous glycemic profile and glycemic variability in patients with obstructive sleep apnea syndrome. Diabetes Res Clin Pract 134:106-112. https://doi.org/10.1016/j.diabres.2017.08.010

28. Byun JI, Cha KS, Jun JE et al (2020) Dynamic changes in nocturnal blood glucose levels are associated with sleep-related features in patients with obstructive sleep apnea. Sci Rep 10(1):17877. https://doi.org/10.1038/s41598-020-74908-x

29. Schmidt S, Christensen MB, Serifovski N et al (2019) Low versus high carbohydrate diet in type 1 diabetes: a 12 -week randomized open-label crossover study. Diabetes Obes Metab 21(7):1680 1688. https://doi.org/10.1111/dom.13725

Publisher's note Springer Nature remains neutral with regard to jurisdictional claims in published maps and institutional affiliations. 